\title{
MODELING STRESS UPTURN AT HIGH STRAIN RATES FOR DUCTILE MATERIALS
}

\author{
Yehuda Partom ${ }^{1, *}$ \\ ${ }^{1}$ Retired from RAFAEL, Israel
}

\begin{abstract}
Ductile materials (mainly metals) exhibit a sharp upturn of stress at strain rates around $10^{3}$ to $10^{4} / \mathrm{s}$, which is not specific to a certain type of material. It is important to consider stress high rate upturn when dealing with high rate loading, such as shock loading and unloading. Using classical strength models, usually calibrated at not so high rates, may lead to errors with high rate loading and not so high pressures. Here we model high rate upturn on the macroscale. We assume that the upturn mechanism is also responsible for the $4^{\text {th }}$ power law mechanism put forward by Swegle and Grady. In the past we calibrated our overstress dynamic viscoplasticity model for aluminium from $4^{\text {th }}$ power law data. Here we use this calibration to predict the high rate stress upturn.
\end{abstract}

\section{Introduction}

Ductile materials (mainly metals) exhibit a sharp upturn of stress at strain rates around $10^{3}$ to $10^{4} / \mathrm{s}$ [1-5], which is not specific to a certain type of material $[5,6]$.

It is important to consider stress high rate upturn when dealing with high rate loading, such as shock loading and unloading. Using classical strength models, usually calibrated at not so high rates, may lead to errors with high rate loading and not so high pressure.

Initially it was believed that stress upturn is caused by a transition from thermal activation of dislocation motion to viscous drag $[7,8]$. But then it was proposed that it is caused by an increase of dislocation generation rate [9].

An entirely different approach to understanding stress upturn is put forward by Fan et al. [6]. They derive their theory analytically on the mesoscale, and verify it by molecular dynamics (MD) simulations. They find that: 1) for high temperature, stress approaches zero (melting), and for very low temperature, stress increases dramatically; 2) strain rate influences stress in an opposite way to that of temperature, similar to timetemperature equivalence of polymers. High rate is equivalent to low temperature, and hence the upturn; and 3) stress upturn results don't depend on details of a specific type of material, which is in general agreement with experimental data.

Here we model stress upturn on the macroscale. First we distinguish between total strain rate and plastic strain rate. In the literature, when plotting stress versus log strain rate, they don't specify which strain rate they mean, total or plastic strain rate. But total strain rate is not a state variable, while effective plastic strain rate may be regarded as a hidden (internal) state variable. Effective plastic strain rate represents the effect of mobile dislocation density and average dislocation velocity, as in Orowan's equation. This leads us to replace the usual $\mathrm{Y}\left(\mathrm{d}_{\mathrm{eff}}\right)$ (flow stress as function of effective deformation rate, or strain rate) equation by the flow curve $\mathrm{d}^{\mathrm{p}}{ }_{\mathrm{eff}}\left(\mathrm{s}_{\mathrm{eq}}{ }^{-}\right.$ $\mathrm{Y}_{\mathrm{qs}}$ ), as in the overstress approach to dynamic viscoplasticity [10].

Next we assume that the high rate upturn mechanism is also responsible for the $4^{\text {th }}$ power law mechanism put forward by Swegle and Grady (SG) [11]. Hints to this effect are made in $[12,13]$. In [14] we calibrated the flow curve of our overstress viscoplastic model to reproduce the $4^{\text {th }}$ power law data for aluminum given in [15]. We show here that the same overstress flow curve that reproduces the $4^{\text {th }}$ power law response reproduces the stress upturn response as well. In section 2 we revisit our $4^{\text {th }}$ power law modeling. In section 3 we use our overstress flow curve to reproduce the high rate stress upturn response.

\section{Revisiting fourth power law response}

Since the works of Barker [15] and Grady [11], it is known that for a steady structured shock in a viscoplastic material, the following power law holds for many materials:

$$
\dot{\varepsilon}_{\text {max }}=\mathrm{d}_{\max }=\mathrm{B}(\Delta \sigma)^{\beta}
$$

where $d_{\max }$ and $\Delta \sigma$ are defined in Fig. 1.

\footnotetext{
Corresponding author: ypartom@netvision.net.il
} 


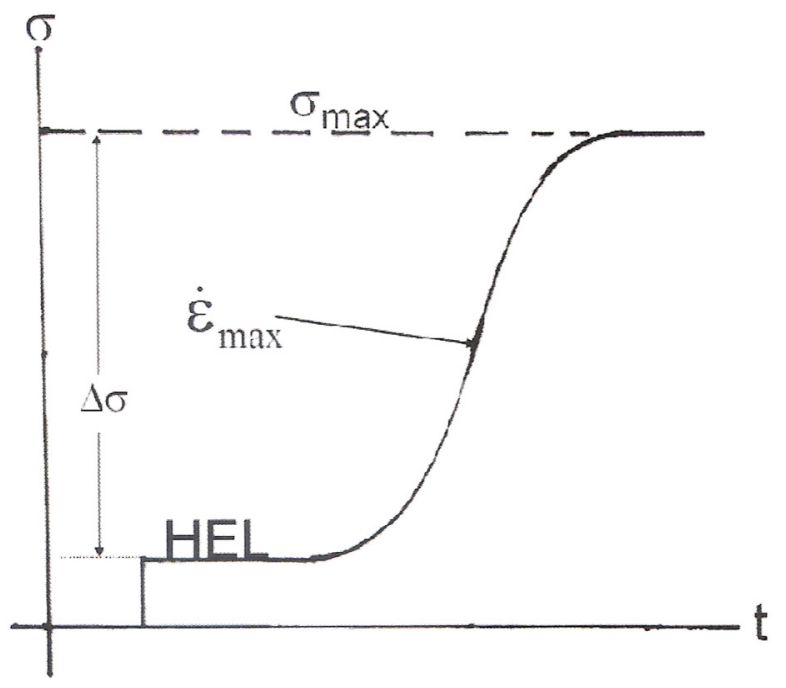

Fig. 1. Schematics of a steady structured viscoplastic wave.

This unique response, known as the $4^{\text {th }}$ power law, is usually shown as straight lines (for different materials) on a log-log plot. In [14] we reproduce the $4^{\text {th }}$ power law response for 6061-T6 aluminum using our overstress approach to dynamic viscoplasticity [10]. We solve there a system of three ODEs describing the steady viscoplastic wave from a planar impact (uniaxial strain), as developed by Duvall and coworkers [16]. The system of ODEs is:

$$
\begin{aligned}
& \dot{\sigma}=\frac{2 G}{c_{L}^{2} / U^{2}-1} d^{p} \\
& \dot{u}=\frac{2 G}{\rho_{0} U\left(c_{L}^{2} / U^{2}-1\right)} d^{p} \\
& \dot{\varepsilon}=\frac{2 G}{\rho_{0} U^{2}\left(c_{L}^{2} / U^{2}-1\right)} d^{p}
\end{aligned}
$$

where $\sigma=$ longitudinal stress component, $\mathrm{u}=$ particle velocity, $\mathrm{U}=$ viscoplastic wave velocity, $\mathrm{c}_{\mathrm{L}}=$ longitudinal sound speed, $\mathrm{G}=$ shear modulus, $\varepsilon=$ longitudinal strain component, $\rho_{0}=$ initial density, $\mathrm{P}=$ pressure and $\mathrm{d}^{\mathrm{p}}=$ longitudinal plastic deformation rate component.

By our overstress approach to dynamic viscoplasticity [10], $\mathrm{d}^{\mathrm{p}}$ is given by:

$$
\mathrm{d}^{\mathrm{p}}=\mathrm{A}\left(\frac{1.5 \mathrm{~s}-\mathrm{Y}_{\mathrm{qs}}}{\mathrm{Y}_{0}}\right)^{\alpha} \mathrm{s}=\sigma-\mathrm{P}
$$

and it can be shown that for uniaxial strain:

$$
\begin{aligned}
& \mathrm{d}_{\mathrm{eff}}^{\mathrm{p}}=\sqrt{\frac{2}{3} \mathrm{~d}_{\mathrm{ij}}^{\mathrm{p}} \mathrm{d}_{\mathrm{ij}}^{\mathrm{p}}}=\mathrm{d}^{\mathrm{p}} \\
& \mathrm{s}_{\mathrm{eq}}=\sqrt{\frac{3}{2} \mathrm{~s}_{\mathrm{ij}} \mathrm{s}_{\mathrm{ij}}}=\frac{3}{2} \mathrm{~s}
\end{aligned}
$$

where $\mathrm{d}^{\mathrm{p}}{ }_{\text {eff }}=$ effective plastic strain rate and $\mathrm{s}_{\mathrm{eq}}=$ equivalent stress. We integrate the system of equations (3) with a standard ODE solver. We use standard material parameters of aluminum, and we run the integration code for different levels of incoming shock. For a given set of the parameters A, $\alpha$ we obtain a straight line on the log$\log$ plot of $\Delta \sigma$ versus maximum strain rate, as in the experiments. Changing these parameters we find that the exponent $\alpha$ controls the slope $\beta$ of this line, and the coefficient A controls its vertical position B. In Fig. 2 we show $\beta$ as function of $\alpha$ from these computations.

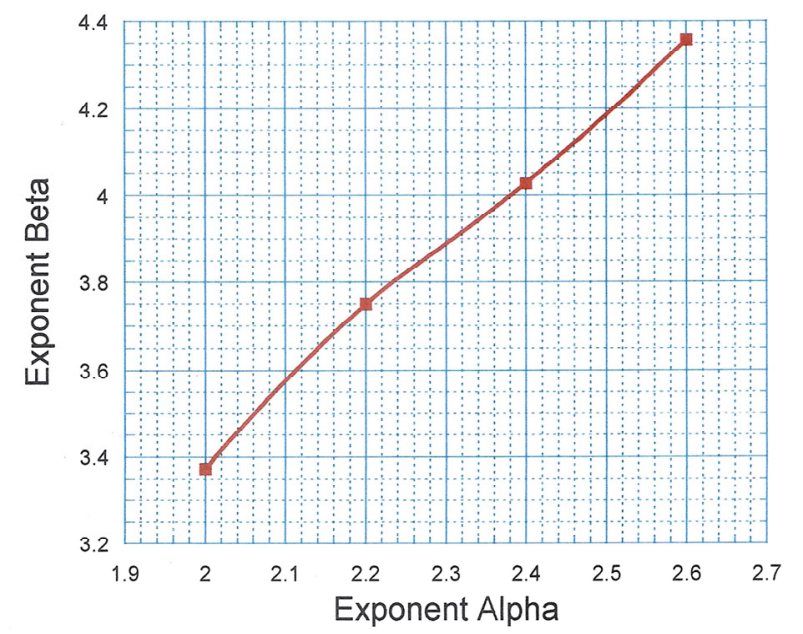

Fig. 2. Exponent $\beta$ (Eq. 1) as function of exponent $\alpha$ (Eq. 3), as obtained by solving the system of equations (2).

We see from Fig. 2 that to get a slope of $\beta=4$ as in the tests, we need to have $\alpha=2.38$. To match the coefficient $B$ for aluminum we use $A=1734 / \mathrm{s}$, which we denote by $d_{0}$, and in Fig. 3 we compare the calculated line of Eq. 1 to the experimental data [15].

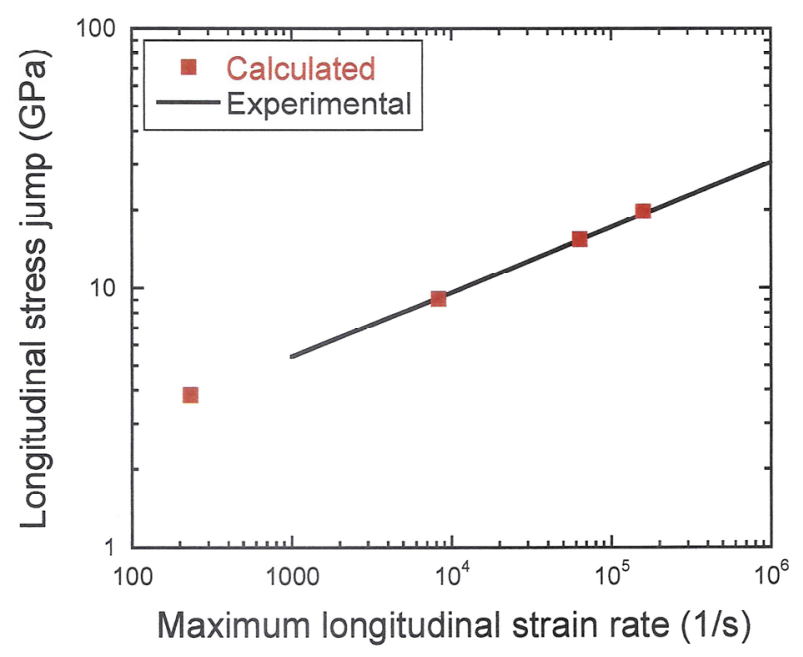

Fig. 3. Calculated versus experimental $4^{\text {th }}$ power plot for 6061 T6 aluminum.

We see from Fig. 3 that the matching is quite good.

\section{Using the calibrated viscoplastic flow curve to predict high rate stress upturn}

As discussed in the introduction, we assume that the same strain rate dependence that controls the $4^{\text {th }}$ power law also drives the stress high rate upturn. We further assume, as indicated in the introduction, that the upturn plot of stress versus log strain rate, should be represented as equivalent stress versus log effective plastic strain rate. 
To show that both, $4^{\text {th }}$ power law and high rate upturn, are caused by the same mechanism, we simply invert Eq. 3 and use Eq. 4 to get:

$$
\mathrm{s}_{\text {eq }}=\mathrm{Y}_{0}\left\lfloor\left(\mathrm{~d}_{\text {eff }}^{\mathrm{p}} / \mathrm{d}_{0}\right)^{1 / \alpha}+1\right]
$$

In Fig. 4 we compare Eq. 5 with upturn data for aluminum from [5].

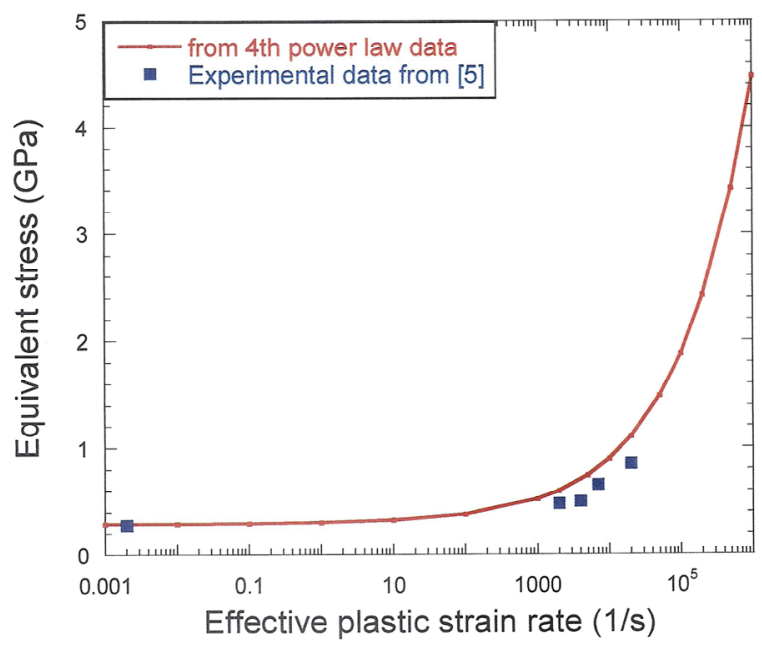

Fig. 4. Equivalent stress upturn plot for aluminum. Comparison of flow curve calculated from $4^{\text {th }}$ power law data with experimental data in [5].

From Fig. 4 we see that:

- The viscoplastic flow curve Eq. 5 is showing the correct upturn behaviour.

- Comparison with experimental data is not too bad, considering the level of scattering in such data.

In Fig. 5 we show the effect of changing $\mathrm{d}_{0}$ or the coefficient $\mathrm{A}$ in Eq. 3. We increase it by 1000 and by $40001 / \mathrm{s}$ (remember that increasing $\mathrm{d}_{0}$ (or A) moves the $4^{\text {th }}$ power line upwards).

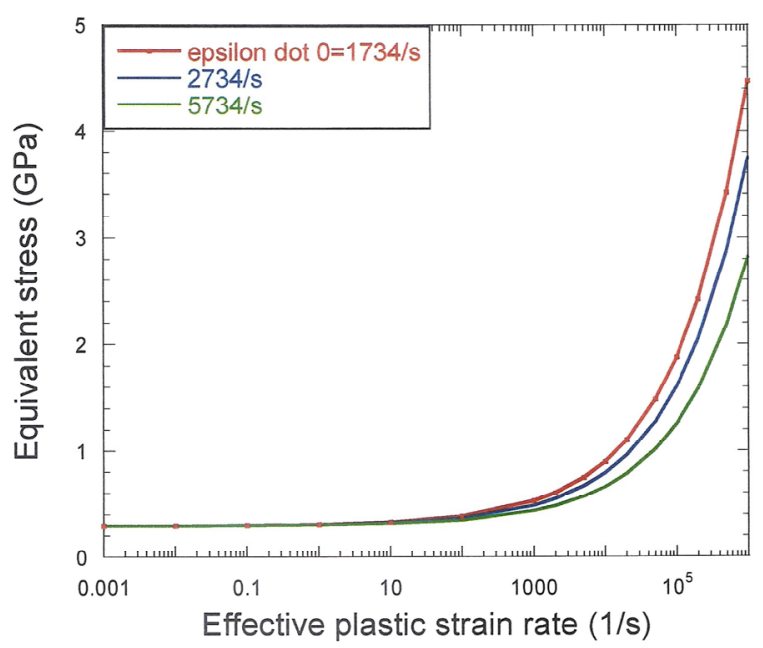

Fig. 5. Equivalent stress upturn plot for aluminum. Influence of $\mathrm{d}_{0}$.

We see from Fig 5 that increasing $\mathrm{d}_{0}$ moves the upturn curve to the right by the same amount. Also, as the computed upturn curve is smooth, it's hard to decide on a specific upturn point. We therefore regard $\mathrm{d}_{0}$ as the upturn strain rate.

In Fig. 6 we show the influence of the flow curve exponent $\alpha$. Although the $4^{\text {th }}$ power law slope and therefore the exponent $\alpha$ are not changing, it's helpful to understand the role of $\alpha$.

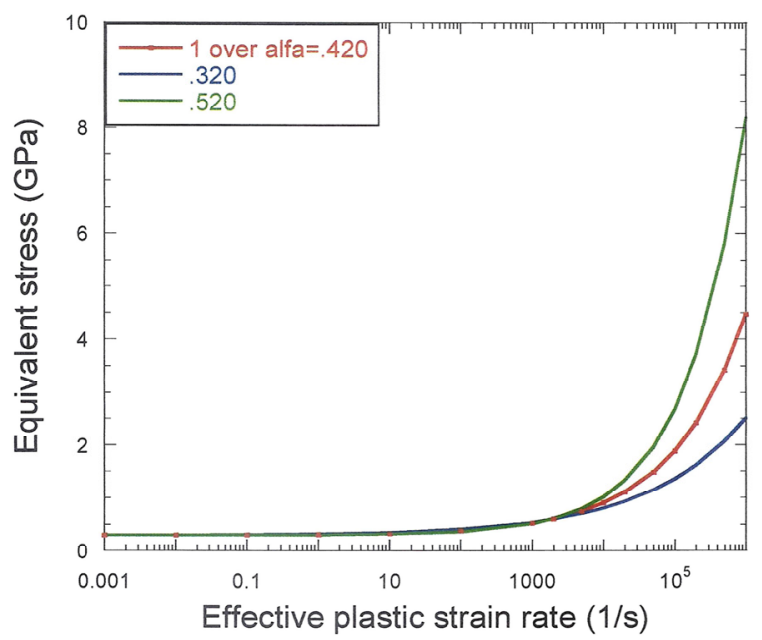

Fig. 6. Equivalent stress upturn plot for aluminum. Influence of the exponent $\alpha$.

We see from Fig. 6 that for high values of $\alpha$, the curve flattens and vice versa. This is because for high $\alpha$, the flow curve (Eq. 3) is steeper, and high stresses are less easily reached.

\section{Summary}

There are two kinds of dynamic tests of ductile materials for which different materials respond in a similar way: 1) planar impact, for which we get a structured viscoplastic steady wave $\left(4^{\text {th }}\right.$ power law response); and 2) uniaxial dynamic loading for which we get a stress upturn as function of $\log$ strain rate at high strain rates (stress upturn response). What is characteristic with these entirely different tests is: 1) they both show similar responses for entirely different materials; and 2) they both involve high strain rates.

In the past we modelled the $4^{\text {th }}$ power law response on the macroscale using our overstress approach to dynamic viscoplasticity. Here we use the flow curve calibrated from a $4^{\text {th }}$ power law test to predict the upturn test response. By doing this we assume that for both tests the high strain rate effect is similar. And indeed according to [6], for high strain rates, dislocations don't have enough waiting time in their potential energy valleys, and their response becomes non-Arrhenius, such that a higher stress is needed to keep them in motion. This is why strain rates affect different materials in a similar way.

\section{References}

1. P.S. Follansbee and U.F. Kocks, A constitutive description of the deformation of copper based on the 
use of the mechanical threshold stress as an internal state variable, Acta Metall. 36, 81-93 (1988)

2. R. Kappor and S. Namet-Nasser, Comparison between high and low strain rate deformation of tantalum, Metall. Mater. Trans. A31, 815-823 (2000)

3. W.S. Lee and T.H. Chen, Rate dependent deformation and dislocation substructure of Al-Sc alloy, Scr. Mater. 5, 1463-1468 (2006)

4. S. Namet Nasser, W.G. Guo and D.P. Kihl, Thermomechanical response of $\mathrm{Al}-6 \mathrm{XN}$ stainless steel over a wide range of strain rates and temperatures, J. Mech. Phys. Solids 49, 1823-1846 (2001)

5. H. Couque, The use of direct impact Hopkinson pressure bar technique to describe thermally activated and viscous drag regimes of metallic materials, Phil. Trans. Royal soc. A372, 20130218 (2014)

6. Y. Fan, Y.N. Osetsky, S. Yip and B. Yildiz, Onset mechanism of strain rate induced flow stress upturn, Phys. Rev. Letters 109, 135503 (2012)

7. P.S. Follansbee and I. Weertman, On the question of flow stress at high strain rates controlled by dislocation viscous flow, Mech. Mater. 1, 345-350 (1982)

8. S. Namet-Nasser and Y. Yulong, Flow stress of FCC polycrystals with application to OFHC $\mathrm{Cu}$, Acta Mater. 46, 565-577 (1998)

9. R.W. Armstrong, W. Arnold and F. Zerilli, Dislocation mechanics of shock induced plasticity, Metall. Mater. Trans. A38, 2605-2610 (2007)

10. Y. Partom, Overstress and flowstress approaches to dynamic viscoplasticity, EPJ web of conferences 94, 04003 (2015)

11. D.E. Grady, Structured shock waves and the fourth power law, J. Appl., Phys. 107, 013506 (2010)

12. M.A. Meyers, Dynamic behavior of materials, John Wiley \& Sons, p. 122 (1994)

13. R.W. Armstrong and S.M. Walley, High strain rate properties of metals and alloys, Int. Mater. Rev. 53, 105-128 (2008)

14. Y. Partom, Calibrating strain rate dependence of viscoplastic flow from fourth power law data, SCCM 2011, AIP conf. Proc. 1426, 1105-1108 (2012)

15. L.M. Barker, Behavior of dense media under dynamic pressure, Gordon and Breech, New York, p. 483 (1968)

16. W. Band and G.E. Duvall, Am. J. Phys. 29, p. 780 (1961) 